\title{
Water filling of hydrophilic nanopores
}

\author{
Ezequiel de la Llave, ${ }^{1}$ Valeria Molinero, ${ }^{2, a)}$ and Damián A. Scherlis ${ }^{1, a)}$ \\ ${ }^{1}$ Departamento de Química Inorgánica, Analítica, y Química Física-INQUIMAE, \\ Facultad de Ciencias Exactas y Naturales, Universidad de Buenos Aires, Ciudad Universitaria, \\ Pab. II, Buenos Aires C1428EHA, Argentina \\ ${ }^{2}$ Department of Chemistry, University of Utah, 315 South 1400 East, Salt Lake City, Utah 84112-0850, USA
}

(Received 12 March 2010; accepted 21 June 2010; published online 21 July 2010)

\begin{abstract}
Molecular dynamics simulations of water in cylindrical hydrophilic pores with diameters of 1.5 and $3 \mathrm{~nm}$ were performed to explore the phase behavior and the nucleation dynamics of the confined fluid as a function of the percentage of volume filled $f$. The interactions of water with the pore wall were considered to be identical to the interactions between water molecules. At low water contents, all the water is adsorbed to the surface of the pore. A second phase consisting of a liquid plug appears at the onset filling for capillary condensation, $f_{\text {onset }}=27 \%$ and $34 \%$ for the narrow and wide pores, respectively. In agreement with experimental results for silica pores, the liquid phase appears close to the equilibrium filling $f_{\text {eq }}$ in the $1.5 \mathrm{~nm}$ pore and under conditions of strong surface supersaturations for the $3 \mathrm{~nm}$ pore. After condensation, two phases, a liquid plug and a surface-adsorbed phase, coexist in equilibrium. Under conditions of phase coexistence, the water surface density $\Gamma_{\text {coex }}$ was found to be independent of the water content and the diameter of the pore. The value of $\Gamma_{\text {coex }}$ found in the simulations $\left(\sim 3 \mathrm{~nm}^{-2}\right)$ is in good agreement with experimental results for silica pores, suggesting that the interactions of water with silica and with itself are comparable. The surface-adsorbed phase at coexistence is a sparse monolayer with a structure dominated by small water clusters. We characterize the density and structure of the liquid and surface phases, the nucleation mechanism of the water plug, and the effect of surface hydrophilicity on the two-phase equilibrium and hysteresis. The results are discussed in light of experiments and previous simulations. (C) 2010 American Institute of Physics. [doi:10.1063/1.3462964]
\end{abstract}

\section{INTRODUCTION}

The development of synthetic routes to build meso- and nanoporous solids of controlled size and structure can be counted among the most celebrated advancements of the past few years in materials chemistry. ${ }^{1-5}$ The unprecedented surface areas $\left(100-1000 \mathrm{~m}^{2} \mathrm{~g}^{-1}\right)$, the uniform diameter of the pores, and their connectivity, plus the possibility of incorporating organic and biological functions, result in an interesting palette of physical and chemical properties which make these systems attractive for a variety of technological uses, ranging from chemical separation and catalysis to optics and microelectronics. ${ }^{1,2}$ Synthetic mesoporous matrices have been based on a broad catalog of inorganic compounds including aluminosilicates, several phosphates, elementary semiconductors, and a variety of inorganic oxides such as titania, silica, and alumina. ${ }^{1-3}$ In particular, hydroxylated mesoporous oxides and silicates exhibit moderate to high hydrophilicity, and so the interactions and the phase behavior of water in these materials have been the target of numerous studies. ${ }^{6-15}$

Sorption-desorption isotherms of water and the associated gas-liquid equilibrium on highly ordered mesoporous solids as MCM-41 and FSM-16 silica and silicates, with pores ranging from 1 to $5 \mathrm{~nm}$, have been characterized ex-

\footnotetext{
a) Authors to whom correspondence should be addressed. Electronic addresses: valeria.molinero@utah.edu and damian@qi.fcen.uba.ar.
}

perimentally by several authors. ${ }^{6-9,11,12,16}$ These isotherms are usually plotted in terms of water content as a function of vapor pressure and typically exhibit three regimes: the surface adsorption plateau, the capillary condensation branchwhere the content of water jumps abruptly-and the final high pressure plateau corresponding to the material mostly filled with liquid. The detailed shape of the curves depends on temperature, hydrophilicity of the walls, and pore size. ${ }^{15}$

A feature of water isotherms on MCM-41 is that adsorption-desorption hysteresis is often observed, i.e., the steep jump in water content during desorption is shifted to lower pressures with respect to adsorption. ${ }^{7,8,11,12}$ Hysteresis in gas sorption experiments is a well-known phenomenon that takes place in pores above certain size (typically larger than $2 \mathrm{~nm}$ in diameter) and is observed only below the hysteresis critical temperature $T_{\text {ch }}$, which depends on the fluidsubstrate pair. In the case of water adsorption experiments, hysteresis has been reported for pores as small as $1.7 \mathrm{~nm}$ in diameter, disappearing for those of $1.4 \mathrm{~nm} ;{ }^{16}$ these sizes are smaller than for most other gases. In spite of these phenomena being known for so long, a microscopic description of the adsorption equilibrium in nanopores, the hysteresis cycle, and its relation to the pore structure are still matter of debate. On the basis of various statistical approaches including Monte Carlo, molecular dynamics, and density functional theory, different authors proposed that in finite cylindrical channels, desorption must occur at the point of equilibrium transition, whereas condensation must be seen somewhere 
between the equilibrium point and the vapor spinodal. ${ }^{17-20}$ In particular, it was argued that the hysteretic behavior of the desorption branch in the simulations was an artifact of the periodic boundary conditions. ${ }^{19}$ These conclusions, however, seem to be at odds with the experimental data presented by Morishige and Ito, who probed nitrogen in several MCM-41 samples to show that neither capillary condensation nor evaporation must necessarily take place at the thermodynamic equilibrium in irreversible isotherms. ${ }^{21}$

The condensation of fluids in confined environments can produce a rich phase diagram with several phase transitions. $^{22,23}$ Computer simulations offer insight into the microscopic structure and processes associated with the phase transformations in nanopores. The formation of a film prior to condensation of argon in cylindrical pores was studied as a function of diameter by Vishnyakov and Neimark, employing the gauge cell Monte Carlo simulation method. ${ }^{24}$ They found that the adsorbed monolayer appeared at lower pressures in narrower pores, yet the density of the monolayer was essentially independent of the pore considered (between 1.4 and $5.5 \mathrm{~nm}$ of diameter). Interestingly, they reported that for the smaller pores (diameters of less than $3 \mathrm{~nm}$ ), the entire surface was filled with the condensed fluid before the second monolayer was formed. In the wider pores, instead, the appearance of a second or even a third layer could be seen prior to capillary condensation. ${ }^{24}$ Based on Monte Carlo simulations in the Gibbs ensemble for TIP4P water confined in featureless Lennard-Jones cylindrical pores, Brovchenko and collaborators $^{23}$ identified up to three kinds of phase transitions in hydrophilic pores aside from the bulklike liquidvapor equilibrium. They classified these three as prewetting, first layering, and second layering transitions, meaning, respectively, vapor-bilayer, vapor-monolayer, and monolayerbilayer coexistence. The predominance of one kind of transition or the other depended on hydrophilicity and pore size. $^{23}$ The use of featureless surfaces in that study does not allow for a clear assignment of one of these scenarios, if any, to the adsorption of water in silica nanopores. In more recent work, Shirono and Daiguji ${ }^{25}$ applied Monte Carlo and molecular dynamics simulations to explore the phase behavior of SPC/E water in silica pores in the range of 1-3 nm of diameter. The silica pores were carved from an atomistic model of $\alpha$-quartz, and hydrogen atoms were added to undercoordinated oxygen atoms, resulting in a surface concentration of hydroxyls of about $2.5 \mathrm{~nm}^{-2}$. These authors characterized three different phases in the pores, consisting of (i) a submonolayer coverage with water molecules exclusively solvating the silanol groups, (ii) a condensed monolayer, and (iii) a completely water-filled pore. The first layering transition informed by Brovchenko et al. ${ }^{23}$ was observed to connect phases (i) and (ii). In addition, they determined that the translational mobility of water in the first adsorption layer was much smaller than the bulk value as consequence of the strong interaction with the surface groups, in agreement with the experimental data reported for silica ${ }^{26}$ and titania. ${ }^{27}$ Even more recently, Mancinelli and co-workers combined neutron diffraction experiments with Monte Carlo simulations to characterize the structure of the pore wall and water in MCM-41 samples $90 \%$ filled. ${ }^{13}$ Different from the crystalline silica structure assumed in Ref. 25, the neutron diffraction and simulations of Ref. 13 indicate that the pore wall is amorphous.

The structure of water in MCM-41 and SBA-15 pores was investigated as a function of water content by Grünberg et al. using magic angle spinning and static solid-state ${ }^{1} \mathrm{H}-\mathrm{NMR}$ spectroscopy. ${ }^{10}$ Their results indicate that all water molecules in the pore participate in hydrogen bonds, either with the $-\mathrm{Si}-\mathrm{OH}$ of the pore wall or with other water molecules. For the MCM-41 pore of $3.3 \mathrm{~nm}$ in diameter, the spectra at low hydration displayed a single line assigned to water bonded to $-\mathrm{Si}-\mathrm{OH}$, which allowed the authors to determine the $-\mathrm{Si}-\mathrm{OH}$ concentration at the pore surface, around $3.5 \mathrm{~nm}^{-2}$. A second line appeared at intermediate hydration that was assigned to water clusters at the surface, exchanging protons with the silanol groups; this line moved toward higher chemical shifts with increasing water content, evidencing an increase in the degree of hydrogen bonding of the surface-adsorbed water molecules. Finally, at 23\% vol/ vol filling, a signal assigned to the formation of a liquid water phase in the partially filled pore appeared at lower chemical shifts. ${ }^{10}$ From the evolution of the NMR spectra with water content, the authors deduced that filling of the MCM-41 pore by water proceeds by adsorption of a monolayer on the surface of the pore, followed by the formation of a liquid water plug that coexists with the surface-adsorbed water. A different mechanism, involving the accumulation of several layers of water on the surface, thickening until they fill the pore, was proposed for the wider $8 \mathrm{~nm}$ diameter SBA-15 pore. ${ }^{10}$

The summary above provides but a partial account of the work done to clarify the filling of hydrophilic cylindrical nanopores. In the present study, we focus on some of the fundamental questions, which remain unsolved, concerning the adsorption of water in hydrophilic mesoporous materials. What is the structure of water inside the pore as a function of filling? Does a liquid monolayer form at the pore interface before a liquid "plug" shows up due to capillary condensation? At what water content does a liquid plug appear? What is the microscopic origin of the hysteresis? Addressing these important questions through simulations requires a molecular model that appropriately represents the hydrogen bonding between the water molecules and the pore wall. The model has to be computationally very efficient to make the simulation of sufficiently long pores possible so as to allow the characterization of the phase transformation in confined water and to afford the lengthy computations necessary not just to equilibrate the water inside the channels but also to evolve the systems for long enough times to obtain insight into the mechanism of nucleation of the liquid plugs.

To address these issues, the present simulations are carried out with the monatomic water $(\mathrm{mW})$ model, ${ }^{28}$ which reproduces the phase structures, the thermodynamic anomalies, and the enthalpy of the phase transitions between liquid, vapor, and ice, with comparable or better accuracy than the most popular atomistic water models. ${ }^{14,28-31}$ Within the $\mathrm{mW}$ model each water molecule is represented by a single particle with three-body interactions that favor tetrahedral "hydrogen-bonded" configurations. $\mathrm{mW}$ involves a computa- 
tional cost two orders of magnitude below that of conventional atomistic models with Ewald sums. The cylindrical pores simulated in this work have an amorphous structure and a relatively smooth surface $\left[t_{90-10}\right.$ width of the pore interface is $1.4 \mathrm{~nm}$ (Ref. 14)]. The smoothness of the interface is in line with the data reported for MCM-41 silica pores in Ref. 32. Nevertheless, the hydrophilic pore wall of the present work is not designed to reproduce the properties of a silica pore but a simpler-more ideal case-in which the interaction of water with the surface moieties is identical to that of water with other water molecules. The rationale of this choice is the similarity in the energies involved in the interaction of water with the hydroxylated groups of a silica surface [predicted by density functional theory (DFT) calculations to be 6.0 and $9.35 \mathrm{kcal} / \mathrm{mol}$ for water as hydrogen donor and acceptor, respectively $\left.{ }^{25}\right]$ and with a surface made entirely of water $\left(8.7 \mathrm{kcal} / \mathrm{mol}\right.$ for water adsorption on ice $\left.{ }^{33}\right)$. We have successfully used this water and pore model to study the freezing, melting, and structure of ice in a $3 \mathrm{~nm}$ diameter pore. ${ }^{14}$ The melting temperature of water in the pore was in excellent agreement with the experiments, suggesting that either the water-wall interactions are not relevant for the calculation of the melting point depression or that - as suggested by the above data-the interactions of water with the water-made walls are similar to that with hydroxylated silica walls. As the liquid-surface-adsorbed (a.k.a. liquid-vapor) equilibrium in the pore may be more sensitive than the liquid-ice equilibrium to the details of the water potential and the water-surface interaction, we compare through the text the results of the coarse-grained simulations with the available experimental data and atomistic simulations for water in silica nanopores to assess whether this simplified model captures qualitatively or quantitatively the properties of water in a silica pore.

Two pore diameters are considered in this work, 1.5 and $3 \mathrm{~nm}$. Hysteresis is not observed in the experimental adsorption curves for the $1.5 \mathrm{~nm}$ silica pore ${ }^{16}$ while it is already quite pronounced for the $3 \mathrm{~nm}$ pore. ${ }^{12,16}$ Using these models we investigate the issues posed above, namely, the clustering of water at the surface of the pore and the formation of the liquid plug as a function of the filling fraction of water. Furthermore, we evaluate the surface coverage at the onset of liquid condensation and when two phases coexist in equilibrium, the effect of the pore-wall interaction energy, and shed light into the nucleation mechanisms controlling the hysteresis cycle.

\section{MODEL AND METHODS}

\section{A. Water force-field}

Water is described by the $\mathrm{mW}$ model, which represents each molecule as a single particle with nonbonding interactions that encourage tetrahedral, "hydrogen-bonded" configurations between water molecules. ${ }^{28}$ The potential energy of $\mathrm{mW}$ is a sum of two- and three-body terms,

$$
\begin{gathered}
E=\sum_{i} \sum_{j>i} \phi_{2}\left(r_{i j}\right)+\sum_{i} \sum_{j \neq i} \sum_{k>j} \phi_{3}\left(r_{i j}, r_{i k}, \theta_{i j k}\right), \\
\phi_{2}\left(r_{i j}\right)=A \varepsilon\left[B\left(\frac{\sigma}{r_{i j}}\right)^{4}-1\right] \exp \left(\frac{\sigma}{r_{i j}-a \sigma}\right), \\
\phi_{3}\left(r_{i j}, r_{i k}, \theta_{i j k}\right)=\lambda \varepsilon\left[\cos \theta_{i j k}-\cos \theta_{0}\right]^{2} \exp \left(\frac{\gamma \sigma}{r_{i j}-a \sigma}\right) \\
\times \exp \left(\frac{\gamma \sigma}{r_{i k}-a \sigma}\right),
\end{gathered}
$$

where $r_{i j}$ is the distance between particles $i$ and $j$, and $\theta_{i j k}$ is the angle subtended by the vectors between the positions of the $i-j$ and $i-k$ pairs of particles, $A=7.049556277, B$ $=0.6022245584, \gamma=1.2, a=1.8$, and $\theta_{0}=109.5^{\circ}$. The value of the parameter $\lambda=23.15$ determines the strength of the tetrahedral interactions, while $\varepsilon=6.189 \mathrm{kcal} / \mathrm{mol}$ and $\sigma$ $=2.3925 \AA$ modulate the strength and characteristic size of water-water interactions, respectively. The absence of electrostatic terms grants the implementation of short cutoffs (all forces vanish at $r_{c}=a \sigma=4.32 \AA$ ), while the lack of hydrogen atoms and the softness of the intermolecular interactions allow for longer integration steps. These features make the $\mathrm{mW}$ potential 180 times computationally more efficient than rigid atomistic models of water using Ewald sums. The $\mathrm{mW}$ model provides a reliable description of the thermodynamic anomalies of bulk water and the structures of different water phases, such as low-density amorphous ice and water clathrates. ${ }^{14,28,29,31}$ Most important for this study, we have recently shown that $\mathrm{mW}$ reproduces the experimental structure of ice in hydrophilic nanopores, its melting temperature, and the existence of a water layer in coexistence with ice at temperatures as low as $50 \mathrm{~K}$ below the melting point. ${ }^{14}$ For an exhaustive assessment of the $\mathrm{mW}$ model, we refer our readers to Refs. $14,28-31$, and 34 .

\section{B. Pore models}

To generate the cylindrical channels for our study, we took instantaneous configurations of liquid water at $298 \mathrm{~K}$ and $1 \mathrm{~atm}$ with cell dimensions of $2.5 \times 2.5 \times 20$ and $5 \times 5$ $\times 10 \mathrm{~nm}^{3}$ as starting building blocks in which we carved out cylindrical pores of 1.5 and $3 \mathrm{~nm}$ in diameter, respectively, with their axis aligned in the $z$-direction. The pore walls were more than $1 \mathrm{~nm}$ wide, well beyond the range of the interactions in the coarse-grained model $(0.432 \mathrm{~nm})$. All water particles belonging to the walls were subjected to the $\mathrm{mW}$ forcefield described above, but, in addition, they interact with their first neighbors through soft harmonic potentials, which preserve the overall structure of the pore while allowing for vibrations around the corresponding equilibrium positions. ${ }^{14}$ For the study of water structure in the pore as a function of water content, the water-wall interactions are described by the same parameters as water-water interaction. In Sec. III C a range of water-wall interaction strengths, $5.6 \leq \varepsilon_{\mathrm{wp}}$ $\leq 6.4 \mathrm{kcal} / \mathrm{mol}$, is considered in order to examine the influence of the hydrophilic character on the filling process and on the density of the surface-adsorbed phase. 


\section{Simulation protocol}

Molecular dynamics simulations were performed using the massively parallel LAMMPS code. ${ }^{35}$ The simulations cells described above were considered with periodic boundary conditions in the three dimensions. The equations of motion were integrated in the canonical $(N V T)$ ensemble using the velocity Verlet algorithm with a time-step of 5 fs. The temperature was controlled using the Nosé-Hoover thermostat with a relaxation time of $0.5 \mathrm{ps}$. All runs were subject to an initial equilibration stage of $10 \mathrm{~ns}$ at $400 \mathrm{~K}$ in order to produce a uniform distribution of water along the pore. This step was followed by a cooling ramp of $2 \mathrm{~K} \mathrm{~ns}^{-1}$ down to $298 \mathrm{~K}$, at which temperature statistical sampling was prolonged for $25 \mathrm{~ns}$, unless otherwise noted. Sampling was conducted for various filling conditions from $10 \%$ to $90 \%$ of the volume of the pore. To assess the reproducibility of the filling percentage that leads to condensation of the liquid phase, we performed three independent simulations for $30 \%$ and $34 \%$ filling in the $3 \mathrm{~nm}$ diameter nanopore. Repetitions were not taken for the $1.5 \mathrm{~nm}$ diameter pore because, as shown in Sec. III, the transition occurs reversibly under equilibrium conditions (i.e., without supersaturation of the surface). The systems contained between 2900 and 7300 water particles accommodated within walls formed by 2925 particles in the case of the narrow pore and 5840 particles in the wide pore. Simulations of the pores at $90 \%$ filling were extended to 200 ns to obtain reliable statistics for the determination of the radial distribution of water. Simulations to investigate the nucleation mechanisms in the pores were evolved for $500 \mathrm{~ns}$.

\section{RESULTS AND DISCUSSION}

\section{A. Phase behavior upon water filling}

The progressive increase of water content in the simulations allowed us to observe the formation of a liquid plug in the pores with the highest water loads. The selected snapshots of Figs. 1 and 2 depict the structure of water inside the 1.5 and $3 \mathrm{~nm}$ diameter pores, respectively, for various filling conditions. In the narrow pore, a single phase, water adsorbed to the surface of the pore, is observed up to $26 \%$ water content. A second phase, a liquid plug, appears at $27 \%$ filling and grows with increasing water content. In the wider pore, the liquid plug forms at $34 \%$ filling. The onset of liquid formation is reproducible in the simulations, which results are in good agreement with the fraction of water at the onset of the capillary adsorption branch of the isotherms for water filling of 1.4 and $2.7 \mathrm{~nm}$ diameter FSM-16 silica pores, about $28 \%$ and $30 \%$, respectively. ${ }^{16}$

Figure 3 shows histograms of the number of water molecules in the narrow pore, binned every $0.5 \AA$ along the pore axis and averaged over $25 \mathrm{~ns}$ trajectories equilibrated at the indicated water contents. The histograms were normalized by the area of the cylinder slice, $0.5 \AA \times 2 \pi \times 7.5 \AA$, resulting in the density profile of water along the pore normalized per unit of pore area. For low concentrations, below 27\% filling, visual inspection of the trajectories for the narrow pore shows that all the water molecules are adsorbed at the surface of the pore (see upper panel of Fig. 1), the surface density profiles are flat (the small fluctuations along the pore

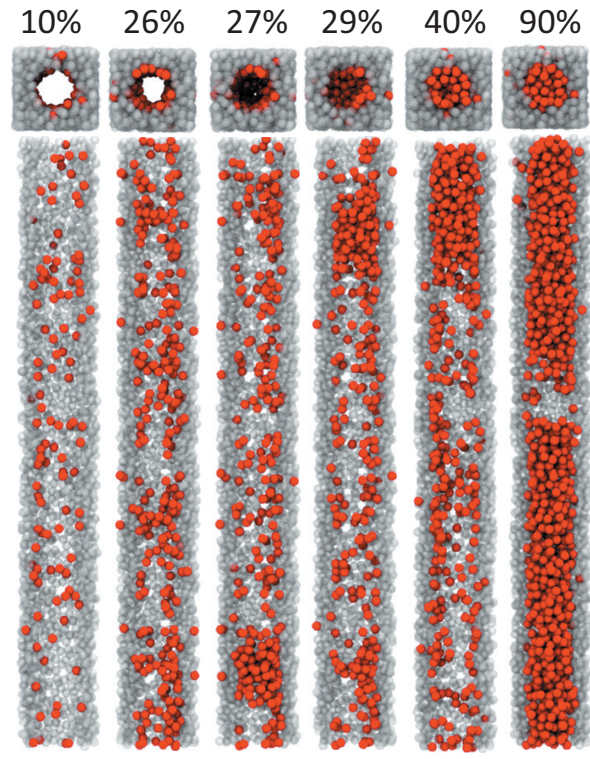

FIG. 1. Snapshots from the $1.5 \mathrm{~nm}$ diameter pore as a function of the water content. Each column corresponds to a different volume fraction of water in the pore (labeled on top). The pores are periodic. The upper panel presents a view of the pore perpendicular to its axis, while the lower panel displays a view parallel to it, in which the front half of the pore was hidden to facilitate the visualization of the water. Molecules of water and pore are represented as solid red and semitransparent gray balls, respectively. Nucleation of the liquid plug occurs under equilibrium conditions at $27 \%$ filling.

seen in Fig. 3 would disappear if the sampling could be extended to hundreds of nanoseconds), and their average yields the density of water at the surface, $\Gamma$. In this regime, before capillary condensation, the surface density increases linearly with the filling percentage $f$,

$$
\Gamma=\frac{N}{A}=\frac{f V \rho}{100 A}=\frac{f \pi R^{2} L \rho}{2 \pi R L 100}=\frac{\rho}{2} \frac{f}{100} R,
$$

where $V$ is the volume, $A$ is the area of the pore channel, $N$ is the number of water molecules contained in it, $f / 100$ is the

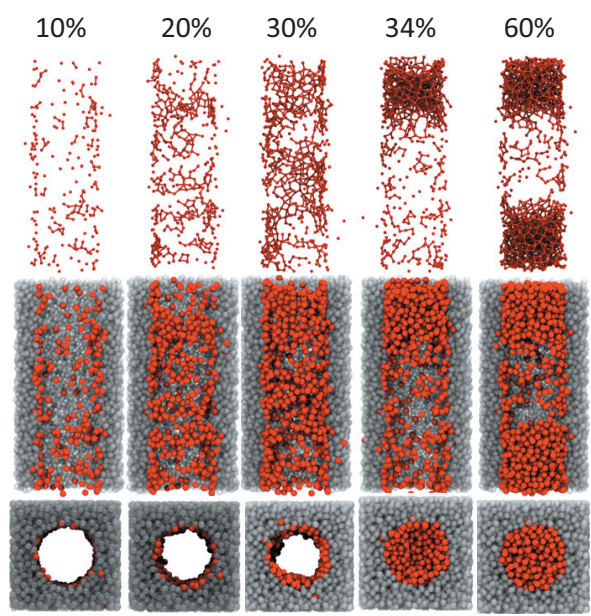

FIG. 2. Snapshots of the $3 \mathrm{~nm}$ diameter pore with increasing water content. The upper panel shows the clustering of the water molecules in the pore (water molecules as small balls; sticks connect molecules within $3.5 \AA$ ). Medium and lower panels show a side and front view, respectively, of the water molecules (red balls) and pore (gray balls). The liquid plug appears at $34 \%$ filling. In equilibrium, the liquid should appear at $\sim 15 \%$ filling. Note the presence of large surface-adsorbed water clusters for $30 \%$ water content. 


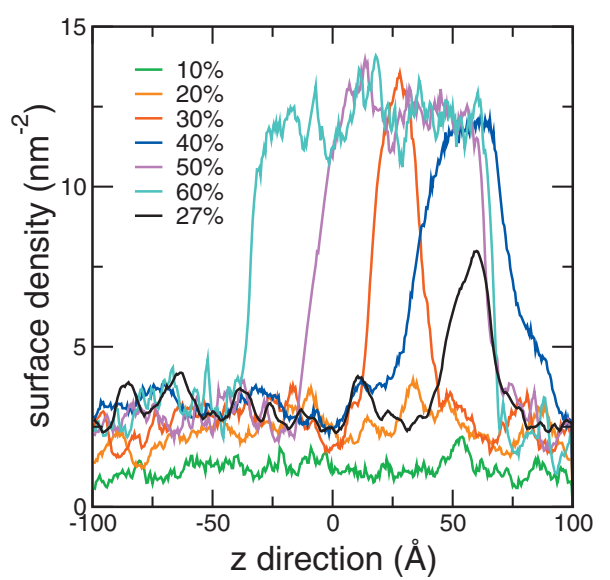

FIG. 3. Density of water per unit area along the axis of the $1.5 \mathrm{~nm}$ diameter pore. Each curve corresponds to a different filling of the pore (labels in the figure). The origin in $z$ is arbitrary, as the pore is periodic. A peak that develops for water contents of $27 \%$ and above corresponds to the liquid plug.

fraction of the pore volume occupied with water, and $\rho$ is the number density of liquid water.

At high water contents, at $f=27 \%$ for the $1.5 \mathrm{~nm}$ pore and $f=34 \%$ for the $3 \mathrm{~nm}$ pore, the symmetry is broken and a region of high density appears in the pore: this indicates the presence of a second phase, consisting of the liquid plug. The snapshots of the $1.5 \mathrm{~nm}$ pore with $26 \%$ (before plug formation) and 29\% filling (after) in Fig. 1 illustrate the differences between the one-phase surface-adsorbed state and the twophase (surface-adsorbed and liquid plug) state of water in the nanopore. Once this plug is formed, the densities of the liquid and surface-adsorbed phases do not experience further changes. In Fig. 3, the liquid plug appears as a region of high density of water per unit area. The increase in pore hydration beyond $27 \%$ filling produces growth of the liquid phase (plug) at the expense of the surface-adsorbed phase, without causing any significant variation in their densities. This situation is analogous to the changes produced by decrease of volume in a bulk system containing liquid and vapor under conditions of thermodynamic equilibrium. To explain the apparent lower density of the water plug for $f=27 \%$ in Fig. 3, it should be noted that in agreement with the experimental results for water in $1.4 \mathrm{~nm}$ diameter FSM-16 silica pores, ${ }^{16}$ we find that the formation of the liquid plug is reversible for the $1.5 \mathrm{~nm}$ diameter pore: at $27 \%$ filling, the plug starts to develop and it alternatively builds up and vanishes along the simulation trajectory resulting in an average density in between the dense liquid and interfacial phases. To observe these oscillations, sampling was conducted over a window of $500 \mathrm{~ns}, 20$ times longer than for the other filling conditions. The hysteresis and nucleation dynamics are discussed below in Sec. III B.

Figure 4 compiles the average surface density of water in the area outside the liquid plug as a function of the water content. Results for both nanopores are included. Note that the equilibrium surface density in the region of two-phase coexistence, above $30 \%$, is around 3 molecules $/ \mathrm{nm}^{2}$ for both the $1.5 \mathrm{~nm}$ and the $3.0 \mathrm{~nm}$ diameter pores. This corresponds to a sparse monolayer coverage. The surface concen-

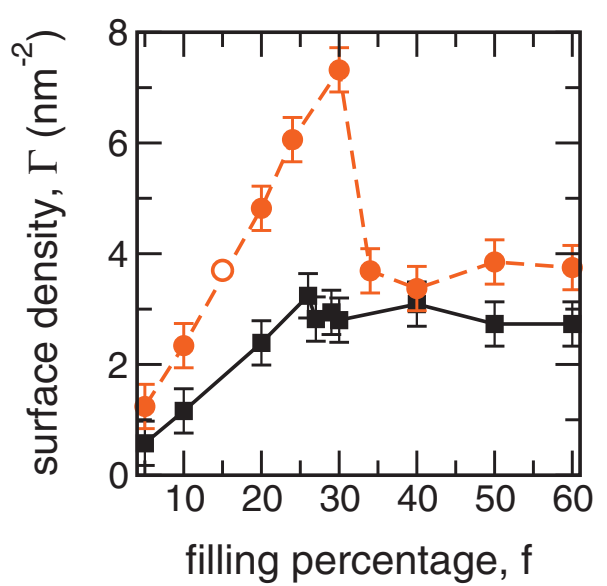

FIG. 4. Density of the surface-adsorbed water phase as a function of filling percentage in the $1.5 \mathrm{~nm}$ (black squares) and $3.0 \mathrm{~nm}$ (red circles) diameter pores. Condensation of the liquid plug occurs at $27 \%$ filling in the $1.5 \mathrm{~nm}$ pore, without surface supersaturation. For the wider pore, the density of water at the surface supersaturates beyond twice the equilibrium value before the liquid plug condenses at $34 \%$ filling. The white-filled red circle indicates the $\Gamma(f)$ for which condensation of the liquid plug should occur under equilibrium conditions.

tration at phase coexistence $\Gamma_{\text {coex }}$ does not appear to have a significant dependence on the pore radius, as also pointed by previous simulations. ${ }^{24}$ This fact suggests that even for the nanoscopic scales of this study, the curvature (i.e., the diameter) of the pore has little effect on the free energy of the water adsorbed at the interface. We note that $\Gamma_{\text {coex }}, \approx 3 \mathrm{~nm}^{-2}$ obtained in the simulations using a pore wall that has the structure and interactions of liquid water is essentially the same as the experimental density of silanol groups at the surface of silica pores: $3.5 \mathrm{~nm}^{-2}$ MCM-41 (Ref. 32) and $3.3 \mathrm{~nm}^{-2}$ for FSM-16. ${ }^{36}$

While the surface density when the two phases coexist in equilibrium, $\Gamma_{\text {coex }}$, is the same for the narrow and wide pores, there is a significant difference in the conditions prior to the nucleation of the liquid plug: Fig. 4 shows that the surface density of water in the wider pore just before capillary condensation $\Gamma_{\text {onset }}$ overshoots to about twice the value of $\Gamma_{\text {coex }}$. The peak in $\Gamma(f)$ around $30 \%$ filling reveals a strong supersaturation of the surface phase before nucleation of the liquid becomes feasible within the time scale of the simulations. This represents a direct contribution to the pore size dependent hysteresis observed in experiments. The maximum surface saturation attained by the $3 \mathrm{~nm}$ diameter pore in the simulations, $\Gamma_{\text {onset }}=7.3 \mathrm{~nm}^{-2}$, is in good agreement with the experimental $6.8 \mathrm{~nm}^{-2}$ reported for a $2.7 \mathrm{~nm}$ FMS-16 silica pore in Ref. 16 but larger than the $4.3 \mathrm{~nm}^{-2}$ observed for a 3 $\mathrm{nm}$ diameter MCM-41 pore in Ref. 12. We note, however, that nucleation is a rare event and with a characteristic time scale that should decrease exponentially with an increase in the excess chemical potential of the liquid phase. Thus, the onset supersaturations at which nucleation of the liquid plug is observed in experiments, where the pores are equilibrated in a time scale of hours, will necessarily be lower than those necessary to trigger a fluctuation that would succeed in forming the liquid in the tens to hundreds of nanoseconds attainable in simulations.

While there is a significant nonequilibrium supersatu- 
rated region in the $3 \mathrm{~nm}$ diameter pore, for the narrower one the supersaturation of the surface-adsorbed phase is negligible and the formation of the plug occurs in equilibrium, without supersaturation, at $27 \%$ filling. Disappearance of hysteresis for water filling of $1.4 \mathrm{~nm}$ silica pores (but not for $2 \mathrm{~nm}$ pores) has been reported in experiments. ${ }^{16}$ With the value of $\Gamma_{\text {coex }}$ at hand, it is straightforward to estimate the water content for which the plug should form under equilibrium conditions for a given pore radius $R$ using Eq. (2) above. In the $3 \mathrm{~nm}$ diameter pore, the equilibrium surface concentration is reached for $f_{\mathrm{eq}} \approx 15 \%$ water content. The fact that the actual water filling percentages $f_{\text {onset }}$ for which the liquid phase appears in experiments ${ }^{12,16}$ do not scale with $R^{-1}$ is a manifestation of hysteresis (formation of metastable supersaturated surface-adsorbed states) on capillary condensation. In early work, the lack of hysteresis in small pores has been attributed to the inability of the fluid to stabilize a meniscus in a channel narrow enough. ${ }^{16,37,38}$ This behavior resembles secondary micropore filling, in contrast to mesoporous filling where hysteresis is necessarily observed. In our simulations, however, no qualitative difference in meniscus formation is observed between the two pore sizes. Experimentally, as confinement increases, hysteresis at room temperature disappears from water isotherms somewhere between 1.4 and $2.0 \mathrm{~nm}$ of diameter. ${ }^{16}$ This critical pore size is smaller for water than for any other adsorbate investigated so far, including $\mathrm{N}_{2}, \mathrm{O}_{2}, \mathrm{Ar}$, or $\mathrm{CO}_{2}{ }^{16,37,39}$ In some of these studies, it has been suggested that the width of the hysteresis loop, or the critical pore diameter, is determined by the molecular size of the adsorbate. ${ }^{37}$

The density of the liquid plug can be computed from the data in Fig. 3 and the radius of the pore. At the core of the fully developed plugs (filling conditions greater than 40\%), densities of 0.971 and $0.988 \mathrm{~g} \mathrm{~cm}^{-3}$ were obtained for the pores of 1.5 and $3 \mathrm{~nm}$, respectively. Recalling that the liquid density of the $\mathrm{mW}$ model at $298 \mathrm{~K}$ is $0.997 \mathrm{~g} \mathrm{~cm}^{-3},{ }^{28}$ these numbers represent a decrease of $3 \%$ and $1 \%$ with respect to the bulk. From sorption isotherms at $\sim 298 \mathrm{~K}$, apparent densities of $0.88-0.89 \mathrm{~g} \mathrm{~cm}^{-3}$ were estimated for capillary condensed water in MCM-41 systems with pores in the range of $3-4 \mathrm{~nm}^{11,39}$ Kocherbitov and Alfredsson ${ }^{11}$ attributed these low values to the dead volume originating in the entrapment of air inside the cavities, suggesting that the microscopic density of the condensed fluid should be closer to that of bulk. More recent measurements by Jähnert et al. determined a 7\% decrease in the density of water confined in cylindrical pores of diameters between 2.5 and $4.4 \mathrm{~nm} .{ }^{12}$ In these experiments, no systematic dependence on the pore width was detected. Figure 5 displays the radial density profile of water in the plug region at a $90 \%$ filling, condition for which the two pores have a well-developed liquid phase. This graph shows that in the case of the wider pore, the distribution of water in the condensed phase is nonuniform also in the radial direction, with a local density of $1.1 \mathrm{~g} \mathrm{~cm}^{-3}$ near the hydrophilic surface, a shallow second peak with maximum density of $1.0 \mathrm{~g} \mathrm{~cm}^{-3}$, and a density close to $0.97 \mathrm{~g} \mathrm{~cm}^{-3}$ at the center of the channel. For the $1.5 \mathrm{~nm}$ diameter pore, the density at the interface is slightly lower, peaking at $1.05 \mathrm{~g} \mathrm{~cm}^{-3}$. For both pore diameters, a marginal amount of

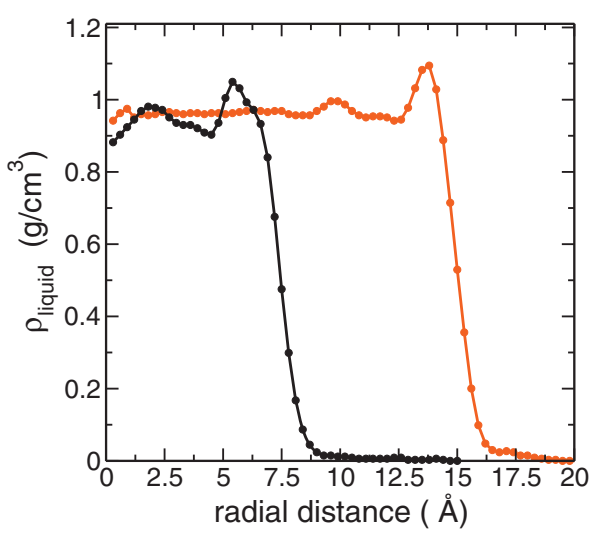

FIG. 5. Radial density profile of water in the liquid plug as a function of the distance to the pore center (the surface-adsorbed region is excluded from these profiles). Profiles for the 1.5 and $3 \mathrm{~nm}$ pore, both for $90 \%$ water filling, are shown in black and red, respectively.

fluid penetrates the pore walls. Atomistic simulations of SPC/E water at $98 \%$ filling in a $1.5 \mathrm{~nm}$ diameter MCM-41 silica pore (modeled with the Vessal et al. potential and Lorentz-Berthelot combination rules for water-silica interactions) predict a slightly larger attraction of water to the pore wall, evidenced by a peak of maximum density of about $1.15 \mathrm{~g} \mathrm{~cm}^{-3}$ near the surface and close to 1 at the center of the pore. ${ }^{40}$ On the other hand, Shirono and Daiguji reported that the density profile of SPC/E water in a fully hydroxylated $1.96 \mathrm{~nm}$ diameter crystalline silica pore with $-\mathrm{Si}-\mathrm{OH} /$ water interactions adjusted from DFT calculations attains a maximum larger than $1.5 \mathrm{~g} \mathrm{~cm}^{-3}$ near the pore wall. ${ }^{25}$ Mancinelli et al. determined the density profile of water in a $90 \%$ filled $1.5 \mathrm{~nm}$ diameter MCM-41 silica pore using reverse Monte Carlo simulation to reproduce neutron diffraction data. ${ }^{13}$ Their calculation yields a strongly inhomogeneous profile: a maximum density close to the surface is around $1.1 \mathrm{~g} \mathrm{~cm}^{-3}$ (comparable to the results of this work and the simulations of Gallo et al. ${ }^{40}$ ) and less than $0.5 \mathrm{~g} \mathrm{~cm}^{-3}$ at the center of the pore. Phase separation, however, was not attained in these Monte Carlo simulations, while the experimental adsorption curves indicate that the $90 \%$ filled pore contains two phases in equilibrium. The lack of phase segregation in the simulations of Ref. 13 is most probably due to the small size of the simulation cell, less than $3 \mathrm{~nm}$ long, and stresses the importance of using large pores, like the $20 \mathrm{~nm}$ length ones of this study, to investigate the distribution of water in partially filled pores. The difficulty in sampling twophase states with small cells is well documented ${ }^{41}$ and makes the study of phase transitions with fully atomistic models computationally very expensive. The inability of a system to phase segregate at low water content should lead to artificially exacerbated heterogeneities in its density profile along the radial direction of the pore. An analysis of the neutron diffraction data with large simulation cells that allow for phase segregation is necessary to accurately determine the actual density profile of water in partially filled silica pores.

\section{B. Hysteresis and nucleation dynamics}

From a thermodynamic standpoint, hysteresis is ascribed to the energetic barriers separating the surface-adsorbed and 


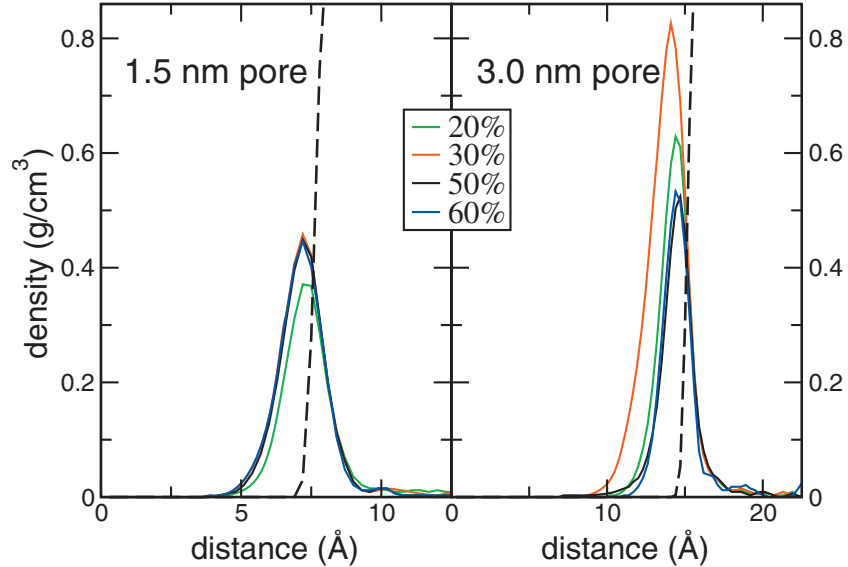

FIG. 6. Radial density profile of surface-adsorbed water as a function of the distance from the center of the pore (the region where there is a liquid plug is excluded from this profile) for several water contents. The left and right panels depict, respectively, the data corresponding to the 1.5 and $3 \mathrm{~nm}$ pores. Dashed black curves show the density profiles for the pore walls.

confined liquid phases. ${ }^{19,24}$ Microscopic correlates of the hysteresis phenomenon have been provided for LennardJones fluids and lattice gas models in idealized pores. ${ }^{17-19} \mathrm{It}$ was suggested that the adsorption mechanism involves the formation of a monolayer of dense gas on the walls, sometimes followed by other layers, with the emergence of density fluctuations along the pore axis ultimately leading to capillary condensation. ${ }^{17,19}$ In this context, the present simulations deliver a rich picture of the process of water filling and the mechanism underlying the hysteresis in adsorption isotherms. Figure 4 indicates that the surface density in the 3 $\mathrm{nm}$ pore doubles the density in the small pore when capillary condensation sets off. The radial density profile of water before and after the point of supersaturation is plotted in Fig. 6 for the large and the small pores in the region excluding the plug. Distinct peaks corresponding to well defined multilayers in smooth-walled pores (see, for example, Refs. 19 and 34) are not visible here. Instead, we see a single peak of increasing width and height up to the concentration corresponding to condensation $f_{\text {onset }}$. Above this hydration, the density profile is invariant in the small pore, while in the larger channel it drops back to the equilibrium value and remains constant thereafter. We recall that equilibrium coverage $\Gamma_{\text {coex }} \approx 3 \mathrm{~nm}^{-2}$ is reached for water content $f \approx 15 \%$ in the $3 \mathrm{~nm}$ pore, whereas at the point of condensation, there is still a single peak in the density profile of the surfaceadsorbed phase but its density is $\Gamma_{\text {onset }}=7.3 \mathrm{~nm}^{-2}$. What is the structure of such denser layer? Some insight into this question can be obtained from Fig. 7, which explores the clustering of the water molecules for different filling fractions. To this end, we classify as clusters the individual groups of connected water molecules, considering a pair of molecules to be connected when they lie at a distance no larger than $3.5 \AA$ - equal to the first minimum in the waterwater radial distribution function in liquid water. ${ }^{28}$ Figure 7 shows the dependence of the number of such clusters on the cluster size (i.e., the number of water molecules contained in the cluster). As the water content is raised, larger clusters tend to form, while the amount of small water networks does
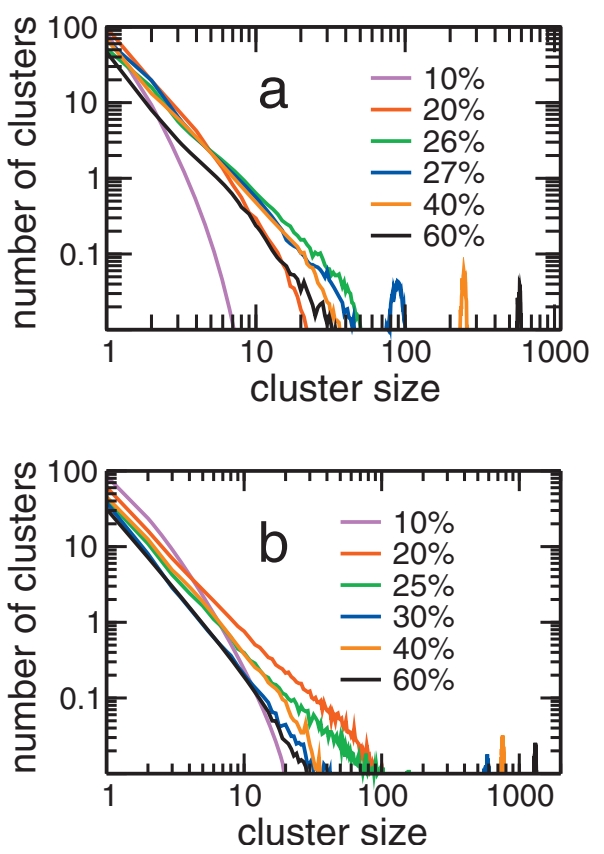

FIG. 7. Logarithmic plot of the size distribution of water clusters for various fillings in (a) the $1.5 \mathrm{~nm}$ pore and (b) the $3 \mathrm{~nm}$ pore. A cluster is a group of connected molecules, considering a pair of water molecules to be connected when they lie no more than $3.5 \AA$ apart (see text). In the narrow pore, a large cluster of about 100 water molecules appears at $27 \%$ filling with the formation of the liquid plug. In the wider pore, a large cluster of more than 600 water molecules appears in the supersaturated surface before condensation of a liquid plug, at $30 \%$ filling.

not vary significantly. A clustering of around 50 molecules marks the critical size to induce plug nucleation in the 1.5 $\mathrm{nm}$ pore. This number increases to $\sim 600$ molecules in the case of the $3 \mathrm{~nm}$ pore. Water condensation is evinced in the sharp peaks appearing on the right part of the plots for fillings above $26 \%$ in the narrow pore or $30 \%$ in the wide pore. We note, however, that in the wider pore, a large isolated cluster with about 180 molecules, beyond the power law distribution, appears already at $25 \%$ filling and grows to more than 600 molecules for 30\% filling (see upper panel of Fig. 2). These large clusters contribute to the thickening of the radial peak of the surface-adsorbed molecules shown in Fig. 6 , but they are not thick enough to bridge the $\sim 1.5 \mathrm{~nm}$ gap at the center of the pore needed to initiate the formation of a plug. Once the plug is formed at higher hydration levels, the cluster size distribution is constant, consistent with a constant value of $\Gamma_{\text {coex }}$ over the two-phase region. The water connectivity of the surface-adsorbed phase remains practically unaffected throughout the two-phase region and quite similar in both pores. Figures 1 and 2 show that the "monolayer" of water is not a compact structure of connected molecules, but rather a collection of small clusters: unconnected water molecules make up $1 / 3$ of the fluid adsorbed at the interface, and clusters with up to five water molecules account for $3 / 4$ of the equilibrium surface coverage. In agreement with the results of the NMR study of Grünberg et al., ${ }^{10}$ we find that adsorption energy of the unconnected water molecules is consistent with them being "hydrogen-bonded" to the pore surface. We measured an average value of $6.83 \pm 0.08 \mathrm{kcal} / \mathrm{mol}$ for the adsorption energy at infinite 


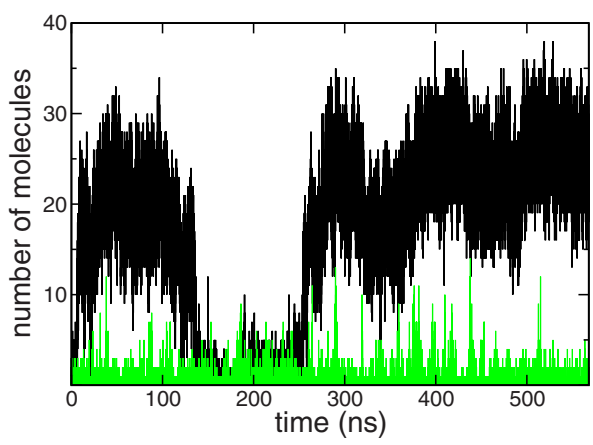

FIG. 8. Time evolution of the number of water molecules beyond the surface layer, in the inner part of the pore, for the $1.5 \mathrm{~nm}$ (black line) and $3 \mathrm{~nm}$ (green line) diameter pores with water content corresponding to the onset of liquid condensation. At $27 \%$ filling the liquid plug forms reversibly for the narrow pore (note the oscillations in the number of water molecules at the center of the pore). There is no reversible plug formation for the wider pore because the liquid plug forms under conditions of strong surface supersaturation. Water does not extend toward the center of the wider pore for $30 \%$ filling, the largest water content before the onset of liquid appearance in the $3 \mathrm{~nm}$ pore.

water dilution at the surface; the value increases to $7.38 \pm 0.12 \mathrm{kcal} / \mathrm{mol}$ after optimization of the energy of the configurations obtained at $298 \mathrm{~K}$ used for the sampling. These adsorption energies of water on the water-wall pore lie right in the average of those computed using DFT for a water molecule interacting with a small piece of silica with a -Si-OH moiety: 6 and $9.35 \mathrm{kcal} / \mathrm{mol}$ for the optimized hydrogen donor and acceptor configurations, respectively. ${ }^{25}$ These results suggest that the good agreement between the water behavior in water-wall pores (both in this study and in Ref. 14) and experiments on MCM-41 silica pores may be due to the similarity in water-wall interaction energies.

Very recently, Kierlik, Puibasset, and Tarjus ${ }^{42,43}$ employed a variety of theoretical tools to investigate the effect of the gas reservoir size on the adsorption isotherms of Lennard-Jones fluids in mesoporous solids. They concluded that by reducing the size of the reservoir, more metastable states of intermediate density become accessible along the sorption-desorption path. ${ }^{42}$ Such metastable states are nonuniform in the pore axis, involving the formation of multiple plugs or "rails." 43 Our present simulations belong to the canonical limit corresponding to a vanishingly small reservoir, and the dimensions of the pores were comparable to those used in Refs. 42 and 43. Nevertheless, the appearance of metastable states consisting of various rails (or, similarly, various bubbles) was never observed for the conditions analyzed in this study. Due to the unfavorable contribution of the surface free energy, important in nanoscopic systems, such states would be higher in free energy than those observed in the molecular dynamics simulations.

We now turn to an analysis of the microscopic fluctuations that succeed in nucleating the liquid plug. Figure 8 depicts the time evolution of the number of particles inside a cylinder of radius equal to $0.4 \mathrm{~nm}$ and concentric with the axis of the narrow pore, for a water concentration of $27 \%$, the onset of liquid plug formation. The radius of this inner cylinder is intended to exclude all surface-adsorbed water molecules and was chosen taking into account the difference between the total radius of the pore and the width of the surface-adsorbed peak in Fig. 6. The massive presence of molecules in the region delimited by this inner cylinder is indicative of the formation of the condensed phase, and so Fig. 8 shows the oscillation of the plug in real time. It becomes apparent that density fluctuations toward the center of the pore are the limiting step for the formation of the plug. According to these simulations, the time scale associated with these oscillations is of the order of $100 \mathrm{~ns}$. The formation of the plug is reversible in the $1.5 \mathrm{~nm}$ diameter pore because, due to the small nucleation barrier, the phase transition occurs under equilibrium conditions. Liquid-vapor oscillations close to or at equilibrium have been reported for water confined in cylindrical and slit hydrophobic nanopores. ${ }^{44-46}$ In the same figure, we present the results for the $3 \mathrm{~nm}$ pore with $30 \%$ water content. For this condition, the adsorbed phase exhibits a single peak with a thickness close to a bilayer, and therefore, in this case we exclude from the sampling all molecules within $6.5 \AA$ from the surface. For the narrow and wide pores at $298 \mathrm{~K}$, we find the same length scale of fluctuations in terms of molecules piling up toward the center of the channel. However, whereas warps two layers thick may suffice to close the gap in the $1.5 \mathrm{~nm}$ pore, longer-ranged fluctuations are necessary as the diameter increases. This is consistent with the interpretation of a different mechanism, based on continuous growing of layers around the surface, for the filling of large $8 \mathrm{~nm}$ diameter SBA-15 pores. $^{10}$

\section{Effect of water-surface affinity}

Within the $\mathrm{mW}$ model, the $\varepsilon$ parameter (equal to 6.189 $\mathrm{kcal} / \mathrm{mol}$ ) modulates the strength of the interaction between particles. $^{28}$ All the results discussed so far were obtained employing the same value of $\varepsilon$ to describe both the interaction of water with the walls of the pore and with itself. To investigate the effect of the water-surface interaction on capillary condensation and hysteresis, we varied the value of $\varepsilon_{\mathrm{wp}}$, determining the water-pore interaction to produce pores with average water adsorption energies from 6 to $7.45 \mathrm{kcal} /$ $\mathrm{mol}$ at $298 \mathrm{~K}$ in the limit of zero surface coverage. For each value of $\varepsilon_{\mathrm{wp}}, 25 \mathrm{~ns}$ molecular dynamics simulations were conducted at various concentrations to localize the conditions of plug formation. Figure 9 summarizes the results found for the $1.5 \mathrm{~nm}$ pore: it can be seen that both the onset filling $f_{\text {onset }}$ needed to unleash the phase transition and the surface density $\Gamma_{\text {onset }}$ at which this occurs are quite sensitive to variations of the interaction energy. As the attraction to the surface is diminished, condensation is observed at smaller water contents. The surface density in equilibrium with the plug $\Gamma_{\text {coex }}$ is also shown to decrease as a function of the adsorption energy. The decrease of $\Gamma_{\text {coex }}$ with diminishing $\varepsilon_{\mathrm{wp}}$ is a direct consequence of the increase in the chemical potential of the adsorbed water derived from a lower attraction of water to the surface, while the chemical potential in the condensed phase should be less affected because the fraction of molecules of the plug in contact with the surface is lower than for the adsorbed phase. In recent atomistic simulations of water in silica nanopores, Shirono and Daiguji ${ }^{25}$ discerned three different phases depending on the chemical 


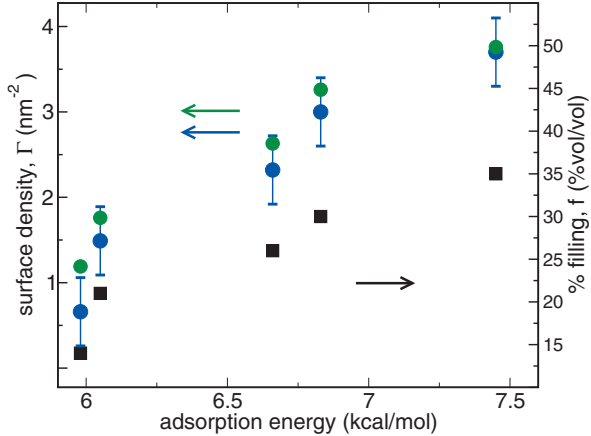

FIG. 9. Surface density of water when liquid plug and surface-adsorbed phase coexist in equilibrium $\Gamma_{\text {coex }}$ (blue circles), onset filling for which the liquid plug appears within $25 \mathrm{~ns}$ of simulation (black squares), and the corresponding onset surface density $\Gamma_{\text {onset }}$ calculated using Eq. (2) (green circles), given as a function of the adsorption energy for water at infinite surface dilution at $298 \mathrm{~K}$.

potential: the first one corresponding to submonolayer coverage, the second one a condensed monolayer, and the third one consisting of a liquid filled cavity. What they classified as condensed monolayer can be identified with the lowdensity surface-adsorbed phase in equilibrium with the liquid plug found in the present study. The maximum surface concentration at coexistence $\Gamma_{\text {coex }}$ attained for the $1.5 \mathrm{~nm}$ pore with this relatively narrow range of interaction parameters is $3.8 \mathrm{~nm}^{-2}$. This is about half the highest surface supersaturation attained in the $3 \mathrm{~nm}$ pore with waterlike wall. This suggests that a much higher attraction to the surface is necessary to reach concentrations for which water multilayers will be formed at the pore wall under equilibrium conditions. These multilayers could form, however, out of equilibrium for wider pores, as reported for the $8 \mathrm{~nm}$ diameter pore by Grünberg et al. ${ }^{10}$ when the barrier to nucleation forces the confined water to states of very high supersaturation.

In the absence of hysteresis, the equilibrium surface density is proportional to the onset filling percentage [Eq. (2)]. In this narrow nanopore, the supersaturation (difference between the onset nonequilibrium $\Gamma_{\text {onset }}$ and two-phase coexistence equilibrium value of the surface density $\Gamma_{\text {coex }}$ ) is relatively small, within $0.4 \mathrm{~nm}^{-2}$. This difference is close to the uncertainty in the simulations and, as we have shown in Sec. III B, disappears when the sampling is extended to hundreds of nanoseconds. These results are in good agreement with the experimental results of Inagaki and Fukushima, which show that the hysteresis in water adsorption and desorption in silica pores disappears in pores of $1.4 \mathrm{~nm}$ diameter. ${ }^{16}$

\section{CONCLUSIONS}

By means of molecular dynamics simulations of water contained in hydrophilic pores at room temperature, it was shown that there is an onset filling of the pore at which a condensed phase develops in the form of a liquid plug. Above this water content, a condensed phase coexists with a low-density phase consisting of water adsorbed on the walls. Further addition of molecules to the system does not alter the densities of the two phases in equilibrium but causes the thickening of the plug. The density of the liquid confined in 1.5 and $3 \mathrm{~nm}$ pores turned out to be, respectively, $3 \%$ and $1 \%$ smaller than that corresponding to bulk water. The equilibrium density of the surface-adsorbed phase corresponded to a sparse monolayer in both pores. In this sparse monolayer, water is hydrogen bonded to the pore but does not form a continuous percolated hydrogen-bonded network with other water molecules; we find that at $\Gamma_{\text {coex }} 3 / 4$ of the water molecules in the surface-adsorbed phase belong to clusters with five or less water molecules.

A qualitative difference found between the 1.5 and $3 \mathrm{~nm}$ pores was that in the latter the surface concentration at the onset of nucleation of the liquid phase was more than twice the equilibrium density. This indicates that a kinetic barrier to condensation, not present in the narrower channel, must be surmounted in the wider pore. We note that water models with longer-ranged interactions could decrease the onset of liquid formation, by exerting attractive forces between water molecules located farther apart. The results of this study are in quantitative agreement with water sorption experiments in mesoporous silica, showing that hysteresis develops in pores of diameters above $\sim 1.5 \mathrm{~nm}$ and that capillary condensation in a $2.7 \mathrm{~nm}$ pore occurs at $f_{\text {onset }} \approx 30 \%$ filling with an associated surface density at the onset of capillary condensation $\Gamma_{\text {onset }}=6.8 \mathrm{~nm}^{-2} .{ }^{16}$ Capillary condensation seems to involve density fluctuations toward the center of the pore, which need to be stronger the wider the pore. At the onset filling, the plug in the narrow pore forms and breaks in an oscillatory fashion with a characteristic time in the range of $100 \mathrm{~ns}$; this reversible behavior is a characteristic of a system at or close to equilibrium conditions. While clusters comprising about 50 water molecules are necessary to develop a plug in the $1.5 \mathrm{~nm}$ diameter pore, a cluster of around 600 molecules was found already in the supersaturated surface-adsorbed phase of the $3 \mathrm{~nm}$ diameter pore at the onset of plug formation.

The onset filling $f_{\text {onset }}$ was found to depend significantly on the water-pore interaction. A decrease in the adsorption energy of only $0.7 \mathrm{kcal} / \mathrm{mol}(10 \%)$ provokes a change of $50 \%$ in $f_{\text {onset }}$, which drops from $\sim 30 \%$ to $\sim 15 \%$. The density of the surface-adsorbed phase is strongly dependent on the hydrophilicity of the surface, at variance with the density of the liquid plug. For the lowest water-pore interactions examined, the surface density in equilibrium with the plug was found to be smaller than the surface density at the point of condensation, suggesting that hysteresis may be more likely to develop in materials with lower water affinity. The reason for this might be related to the kinetic barriers associated with liquid condensation from low-coverage phases. Further analysis would be required to corroborate this possibility.

\section{ACKNOWLEDGMENTS}

This study has been supported by a collaborative grant of the Agencia Nacional de Promoción Científica y Tecnológica de Argentina, PICT 2007-2111 (V.M. and D.A.S.), and by the Beckman Young Investigator Program (V.M.). E.d.l.L. acknowledges CONICET for a doctoral fel- 
lowship. We thank the Center of High Performance Computing of the University of Utah for the allocation of computing time.

${ }^{1}$ C. Kresge, M. Leonowicz, W. Roth, and J. Vartuli, Nature (London) 359, 710 (1992).

${ }^{2}$ M. Davis, Nature (London) 417, 813 (2002).

${ }^{3}$ G. J. d. A. A. Soler-Illia, C. Sanchez, B. Lebeau, and J. Patarin, Chem Rev. (Washington, D.C.) 102, 4093 (2002).

${ }^{4}$ J. Lee, M. C. Orilall, S. C. Warren, M. Kamperman, F. J. Disalvo, and U. Wiesner, Nature Mater. 7, 222 (2008).

${ }^{5}$ G. Férey, Chem. Soc. Rev. 37, 191 (2008).

${ }^{6}$ P. Llewellyn, F. Schüth, Y. Grillet, F. Rouquerol, and K. Unger, Langmuir 11, 574 (1995).

${ }^{7}$ S. Takahara M. Nakano, S. Kittaka, Y. Kuroda, T. Mori, H. Hamano, and T. Yamaguchi, J. Phys. Chem. B 103, 5814 (1999).

${ }^{8}$ P. Smirnov, T. Yamaguchi, S. Kittaka, S. Takahara, and Y. Kuroda, J. Phys. Chem. B 104, 5498 (2000).

${ }^{9}$ J. Oh, W. Shim, J. Lee, J. Kim, H. Moon, and G. Seo, J. Chem. Eng. Data 48, 1458 (2003).

${ }^{10}$ B. Grünberg, T. Emmler, E. Gedat, J. Shenderovich, G. Findenegg, H. Limbach, mand G. Buntkowsky, Chem.-Eur. J. 10, 5689 (2004).

${ }^{11}$ V. Kocherbitov and V. Alfredsson, J. Phys. Chem. C 111, 12906 (2007).

${ }^{12}$ S. Jähnert, F. Vaca Chávez, G. E. Schaumann, A. Schreiber, M. Schönhoff, and G. H. Findenegg, Phys. Chem. Chem. Phys. 10, 6039 (2008).

${ }^{13}$ R. Mancinelli, S. Imberti, A. K. Soper, K. H. Liu, C. Y. Mou, F. Bruni, and M. A. Ricci, J. Phys. Chem. B 113, 16169 (2009).

${ }^{14}$ E. B. Moore, E. de la Llave, K. Welke, D. A. Scherlis, and V. Molinero, Phys. Chem. Chem. Phys. 12, 4124 (2010).

${ }^{15}$ E.-P. Ng and S. Mintova, Microporous Mesoporous Mater. 114, 1 (2008).

${ }^{16}$ S. Inagaki and Y. Fukushima, Microporous Mesoporous Mater. 21, 667 (1998).

${ }^{17}$ G. Heffelfinger, F. van Swol, and K. Gubbins, J. Chem. Phys. 89, 5202 (1988).

${ }^{18}$ U. Marini Bettolo Marconi and F. Van Swol, Phys. Rev. A 39, 4109 (1989).

${ }^{19}$ A. Papadopoulou and F. van Swol, J. Chem. Phys. 97, 6942 (1992).

${ }^{20}$ A. Neimark, P. Ravikovitch, and A. Vishnyakov, Phys. Rev. E 62, R1493 (2000)

${ }^{21}$ K. Morishige and M. Ito, J. Chem. Phys. 117, 8036 (2002).

${ }^{22}$ P. Tarazona, U. M. Marconi, and R. Evans, Mol. Phys. 60, 573 (1987).
${ }^{23}$ I. Brovchenko, A. Geiger, and A. Oleinikova, J. Chem. Phys. 120, 1958 (2004).

${ }^{24}$ A. Vishnyakov and A. Neimark, J. Phys. Chem. B 105, 7009 (2001).

${ }^{25}$ K. Shirono and H. Daiguji, J. Phys. Chem. C 111, 7938 (2007).

${ }^{26}$ S. Takahara, N. Sumiyama, S. Kittaka, T. Yamaguchi, and M. BellissentFunel, J. Phys. Chem. B 109, 11231 (2005).

${ }^{27}$ E. Mamontov, D. J. Wesolowski, L. Vlcek, P. T. Cummings, J. Rosenqvist, W. Wang, and D. R. Cole, J. Phys. Chem. C 112, 12334 (2008).

${ }^{28}$ V. Molinero and E. B. Moore, J. Phys. Chem. B 113, 4008 (2009).

${ }^{29}$ E. B. Moore and V. Molinero, J. Chem. Phys. 130, 244505 (2009).

${ }^{30}$ R. C. DeMille and V. Molinero, J. Chem. Phys. 131, 034107 (2009).

${ }^{31}$ L. C. Jacobson, W. Hujo, and V. Molinero, J. Phys. Chem. B 113, 10298 (2009).

${ }^{32}$ I. G. Shenderovich, D. Mauder, D. Akcakayiran, G. Buntkowsky, H.-H. Limbach, and G. H. Findenegg, J. Phys. Chem. B 111, 12088 (2007)

${ }^{33}$ A. Pelmenschikov and H. Ogasawara, J. Phys. Chem. A 106, 1695 (2002).

${ }^{34}$ N. Kastelowitz, J. C. Johntson, and V. Molinero, J. Chem. Phys. 132, 124511 (2010).

${ }^{35}$ S. J. Plimpton, J. Comput. Phys. 117, 1 (1995).

${ }^{36}$ T. Ishikawa, M. Matsuda, A. Yasukawa, K. Kandori, S. Inagaki, T. Fukushima, and S. Kondo, J. Chem. Soc., Faraday Trans. 92, 1985 (1996).

${ }^{37}$ P. Llewellyn, Y. Grillet, F. Schüth, and H. Reichert, Microporous Mater. 3, 345 (1994).

${ }^{38} \mathrm{~J}$. Gregg, in Adsorption at the Gas Solid and Liquid Solid Interface, edited by F. Rouquerol, K. S. W. Sing, and H. Kral (Elsevier, Amsterdam, 1988).

${ }^{39}$ P. J. Branton, M. Hall, K. S. Treguer, and K. S. W. Sing, J. Chem. Soc., Faraday Trans. 91, 2041 (1995).

${ }^{40}$ P. Gallo, M. Rovere, and S.-H. Chen, The Journal of Physical Chemistry Letters 1, 729 (2010).

${ }^{41}$ K. Binder, J. Horbach, W. Kob, W. Paul, and F. Varnik, J. Phys.: Condens. Matter 16, S429 (2004).

${ }^{42}$ E. Kierlik, J. Puibasset, and G. Tarjus, J. Phys. Condens. Matter 21, 155102 (2009).

${ }^{43}$ J. Puibasset, E. Kierlik, and G. Tarjus, J. Chem. Phys. 131, 124123 (2009).

${ }^{44}$ G. Hummer, J. C. Rasaiah, and J. P. Noworyta, Nature (London) 414, 188 (2001).

${ }^{45}$ O. Beckstein and M. Sansom, Proc. Natl. Acad. Sci. U.S.A. 100, 7063 (2003).

${ }^{46}$ L. Xu and V. Molinero, J. Phys. Chem. B 114, 7320 (2010). 